\title{
Multispectral Image Reproduction via Color Appearance Mapping
}

\author{
Ying Wang, Sheping Zhai and Zhongmin Wang \\ School of Computer Science \& Technology, Xi'an Univ. of Posts \& \\ Telecommunications, Xi'an China \\ wangyingjsj@xupt.edu.cn
}

\begin{abstract}
To achieve the color consistent reproduction of multispectral images in the different viewing condition, a new method of multispectral image reproduction via color appearance mapping was proposed. Firstly, through the introduction of color appearance transformation, the color appearance description of the source spectral reflectance in the source viewing condition was obtained. Then by the construction of the inverse model, the high dimension spectra in the destination viewing condition were evaluated, which was color appearance matching but spectral mismatching with the source image. Finally, to improve the spectral precision of the reproduced spectra, the evaluated spectra were corrected by the method of metamerism correction based on the source spectra, and then the reproduced spectral image was obtained, which matched the source image in color appearance and in spectra when the reproduction viewing condition was different from the source. Experiments show that the perceptual color difference and the spectral error between the reproduced multi-spectral image and its original in the different viewing condition are small. The new method preserves the spectral information of the source multispectral image and achieves equal perceptual reproduction to the source image.
\end{abstract}

Keywords: multispectral image reproduction, color appearance mapping, spectral evaluation, metamerism correction, viewing condition independent space

\section{Introduction}

Multi-spectral images are those whose pixel values are spectral reflectance of source scenes. They are mainly used for the accurate and consistent color reproduction of source scenes under different illuminant. Now they have been widely used in high-end imaging fields such as art archiving [1-2], medicine [3-4], military target imaging.

Current research on multispectral image reproduction mainly focuses on accurately reproducing the spectral reflectance of the source scene objects. It aims at achieving the real color perception of the source scenes in various viewing conditions. However, in many applications, the consistent color appearance reproduction of multispectral images is required when the reproduction viewing condition is different from the source condition.

To achieve the consistent color reproduction of the multispectral image in different viewing condition, an algorithm of multispectral image reproduction via color appearance mapping is presented. By introducing the color appearance transformation, the spectral reflectance of the multispectral image is transformed from the spectral reflectance space to the viewing condition independent space. Then by constructing the inverse transformation, the spectral reflectance of the image is reproduced in the destination viewing condition. Meanwhile, in order to keep the source spectral information as more as possible, the 
evaluated spectra are corrected based on the source spectra by using a metamerism correction method. From this, the reproduction multi-spectral image is achieved whose color appearance is consistent and spectra are approximating to the source image.

\section{Algorithm Framework}

The data of multi-spectral images are the spectral reflectance of the image scene in the range of visible light. They can reflect the most essential information of the image color. Thus the color reproduction accuracy of the image in a specific viewing condition is high. However, the spectral color space is viewing-condition dependent space, that is, for the same spectral values; the image color perceived by the human eye is changed with the change of viewing-condition. During the reproduction of the multispectral images, the reproduction viewing-condition often is different from the source viewing-condition. Thus achieving the same color appearance when the viewing-condition is changed is an important requirement.

The algorithm framework in this paper is shown in Figure 1. Firstly, multispectral images are transformed from the spectral color space to the CIE color space. CIE color space also is the viewing-condition dependent space. Thus the image is then transformed from the CIE space to the JCH viewing-condition independent space by color appearance transformation based on the source viewing-condition. Then the color appearance inverse transformation is implemented based on the destination viewing-condition and the chroma values $X^{\prime} Y^{\prime} Z^{\prime}$ in the CIE color space are achieved. Finally, by the chroma inverse transformation, the chroma values $X^{\prime} Y^{\prime} Z^{\prime}$ are transformed to the spectral space and the reproduction multispectral image is obtained. The chroma inverse transformation includes high-dimension spectral evaluation and metamerism correction. We will discuss it in 3.3.

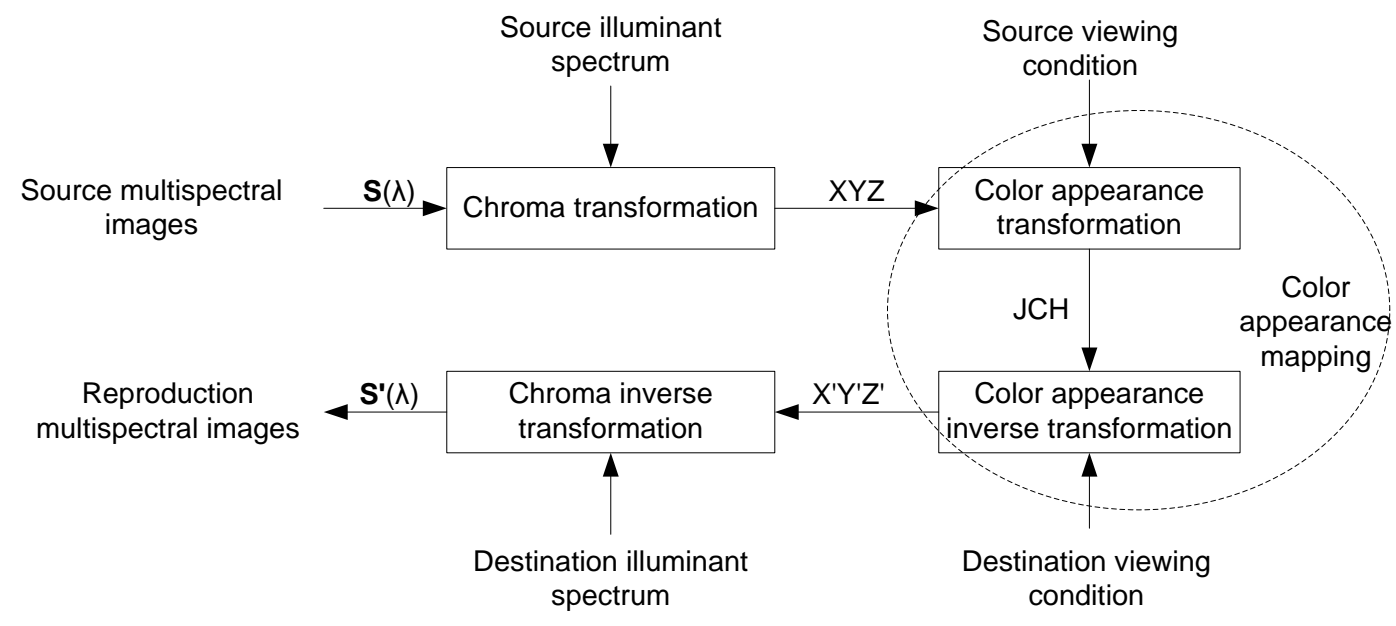

Figure 1. Algorithm Framework of our Approach

\section{Multi-Spectral Image Reproduction Via Color Appearance Mapping}

\subsection{Chroma Transformation}

Multi-spectral images are acquired by narrow-band sampling in the range of visible light, e.g. from $400 \mathrm{~nm}$ to $700 \mathrm{~nm}$. So the spectral reflectance is high-dimension data. Given a 
spectral reflectance $s$ of one pixel in the source image, the CIEXYZ tristimulus value $t$ of this pixel in source viewing-condition is

$$
\boldsymbol{t}=\left(\begin{array}{lll}
X & Y & Z
\end{array}\right)^{T}=k \cdot \boldsymbol{F} \cdot \operatorname{diag}(\boldsymbol{E}) \cdot \boldsymbol{s}
$$

where $k=\boldsymbol{F}_{v} \cdot \operatorname{diag}(\boldsymbol{E}) \bullet$ ones is a constant which can maintain the tristimulus $Y=100$ on the complete diffuse surface. $\boldsymbol{F}$ is the standard observer Color Matching Function. $\boldsymbol{F}_{y}$ is the $y$-component in the Color Matching Function. $\boldsymbol{E}$ is the spectral power distribution of the source illuminant. $\operatorname{diag}(\bullet)$ Means generating a diagonal matrix by using a vector as its diagonal elements. Ones is a vector that all its elements are 1 and its dimension is the same as $\boldsymbol{s}$.

\subsection{Color Appearance Mapping}

CIE color space can only express the color perceptual of a person with normal color vision under a specific light [5]. When the destination viewing-condition is the same as the source, CIEXYZ chroma values are equal means the color is matching in these two scenes. Otherwise the color perceptual of human eyes will be different. Therefore, the CIE space is view-condition dependence. In order to achieve the color appearance consistence of the multispectral images when the destination viewing-condition is different form the source, the color values must be matching in the viewing-condition independence space. To descript the color appearance mapping process, we use $\Omega_{\text {CIE }}$ to denote CIE color space, $\Omega_{\text {ICH }}$ to denote viewing-condition independence space, and define the color appearance transformation as $j_{s}=C A M_{s r_{-} v c}\left(t_{s}\right), \boldsymbol{t}_{s} \in \boldsymbol{\Omega}_{C I E}, \boldsymbol{j}_{s} \in \boldsymbol{\Omega}_{J C H}$, where $s r c_{-} v$ is the source viewing-condition. We also define the color appearance inverse transformation as $\boldsymbol{t}_{s}{ }^{\prime}=C A M^{-1}{ }_{d s_{-} v c}\left(\boldsymbol{j}_{s}\right), \boldsymbol{t}_{s}{ }^{\prime} \in \boldsymbol{\Omega}_{C I E}$, where $d s t_{-} v c$ is the destination viewing-condition. Thus the process of color appearance mapping is expressed as

$$
\boldsymbol{t}_{s}{ }^{\prime}=\left(\begin{array}{lll}
X^{\prime} & Y^{\prime} & Z^{\prime}
\end{array}\right)^{T}=C A M_{d s t_{-} v c}^{-1}\left(C A M_{s r c_{-} v c}\left(\boldsymbol{t}_{s}\right)\right)
$$

The color appearance model can predict the color appearance values in various viewing-condition [5]. In this paper we use international standard color appearance model CIECAM02 [6] to calculate the color appearance values in source viewingcondition, that is, to accomplish the color appearance transformation. Then we use CIECAM02 inverse model to calculate the color values under destination viewingcondition, that is, to accomplish the color appearance inverse transformation. The calculating process of CIECAM02 model is described in [6].

\subsection{Chroma Inverse Transformation}

The colorimetric values obtained by color appearance mapping must be exerted chroma inverse transformation on to get the spectral reflectance. In the nature, the different objects with different spectra characteristics can present the same color in the specific illuminant, that is, they have the same colorimetric values. Therefore, even the colorimetric values and the viewing condition are known, the high-dimension spectral reflectance cannot be gained by the simple inverse operation of Equation (1). It needs to design an algorithm. The chroma inverse transformation algorithm proposed in this paper includes high-dimension spectrum evaluation and metamerism correction. 
3.3.1. High-dimension spectrum evaluation: Through normalizing the colorimetric values $t_{s}$ ' obtained by equation (2), we can calculate the normalized tristimulus values c,

$$
\boldsymbol{c}=\left[\begin{array}{lll}
X_{\text {Norm }} & Y_{\text {Norm }} & Z_{\text {Norm }}
\end{array}\right]^{T}=\left[\begin{array}{lll}
\frac{X^{\prime}}{X_{N}} & \frac{Y^{\prime}}{Y_{N}} & \frac{Z^{\prime}}{Z_{N}}
\end{array}\right]^{T}
$$

where $\left[\begin{array}{ccc}X_{N} & Y_{N} & Z_{N}\end{array}\right]^{T}=k \square^{F} \square \operatorname{diag}\left(\boldsymbol{E}^{\prime}\right) \square_{\text {ones }}, \boldsymbol{E}^{\prime}$ is the spectral power distribution of the destination viewing illuminant.

Now we use $s^{\prime}$ to represent the spectral reflectance calculated by the CIE chroma values $t_{s}{ }^{\prime}$, then the spectral reflectance estimated by the normalized tristimulus values can be represented as

$$
s^{\prime}=H c
$$

where $\boldsymbol{H}$ is the evaluation matrix.

To calculate the matrix $\boldsymbol{H}$, we select a set of spectral reflectance samples, and denote as $\left\{s_{k}\right\}, 1 \leq k \leq M_{q}$, where $M_{q}$ is the number of the samples. By Equation (1) to (3), we can gain the normalized tristimulus value set in the reproduction viewing condition and denote it as $\left\{\boldsymbol{c}_{k}\right\}, 1 \leq k \leq M_{q}$. Then the transformation from the spectral reflectance sample set to the normalized tristimulus values can be established as

$$
C=D S
$$

where $C=\left[\begin{array}{llll}c_{1} & c_{2} & \ldots & c_{M_{q}}\end{array}\right], S=\left[\begin{array}{llll}s_{1} & s_{2} & \ldots & s_{M_{q}}\end{array}\right]$, and matrix $\boldsymbol{D}$ can be denoted as

$$
\boldsymbol{D}=\boldsymbol{N} \square \boldsymbol{F} \square \operatorname{diag}\left(\boldsymbol{E}^{\prime}\right)
$$

$$
N=\operatorname{diag}\left(\left\lfloor\frac{k}{X_{N}} \frac{k}{Y_{N}} \frac{k}{Z_{N}}\right]^{T}\right)
$$

Based on the Equation (4), the transformation from the normalized tristimulus value set to the spectral reflectance sample set can be denoted as

$$
S=\boldsymbol{H} C
$$

By Equation (5) and (8), we know that the matrix $\boldsymbol{H}$ is the pseudoinverse matrix of $\boldsymbol{D}$. Multiplying $\boldsymbol{C}^{T}\left(\boldsymbol{C} \boldsymbol{C}^{T}\right)^{-1}$ to both sides of the equation (8), we get

$$
S \boldsymbol{C}^{T}\left(\boldsymbol{C} \boldsymbol{C}^{T}\right)^{-1}=\boldsymbol{H} \boldsymbol{C} \boldsymbol{C}^{T}\left(\boldsymbol{C} \boldsymbol{C}^{T}\right)^{-1}
$$

Thus

$$
\boldsymbol{H}=\boldsymbol{S} \boldsymbol{C}^{T}\left(\boldsymbol{C} \boldsymbol{C}^{T}\right)^{-1}
$$

Now by using Equation (4), we can calculate the high-dimension spectral reflectance $s^{\prime}$ from CIE chroma value $t_{s}{ }^{\prime}$.

3.3.2. Metamerism correction: The reproduction of multi-spectral images usually pursues the target that the spectra of the reproduction images are consistent to that of the source. Since the spectrum is independent of the human visual system perception for color, and the colorimetric values cannot reflect the spectra information of the image color, only the spectral matching or chroma matching cannot guarantee the quality of color reproduction of the multispectral image in different viewing condition. The algorithm described in 3.3.1 can achieve the color appearance matching by the color appearance transformation in the source side and the inverse transformation in the reproduction side. However, the spectral matching and color appearance matching are all critical to the quality of the image reproduction. Thus, we exert the metametrism 
correction to the spectral reflectance calculated by Equation (4) to achieve the spectral matching.

Metamerism makes one tristimulus value corresponding to infinite spectral reflectance. In Equation (8), the matrix $\boldsymbol{H}$ is the pseudoinverse of $\boldsymbol{D}$. It results in that the spectral reflectance $s$ ' calculated by the normalized tristimulus value $c$ in Equation (4) is just one of a set of metamerism spectrum in reproduction viewing condition. It matches the source spectrum $s$ in color appearance, but mismatches in spectrum. To match or approximate the source spectrum, the estimated spectrum $s^{\prime}$ must be corrected to achieve its reproduction $s_{r}$, which is metameric with $s^{\prime}$ under reproduction viewing condition and has the minimum spectral error with the source spectrum $s$. So we define

$$
s_{r}=M C\left(s^{\prime}, s\right)
$$

Where $M C(\square)$ is the metamerism correction function.

In 1953, Wyszecki proposed a hypothesis [7] that any color stimuli can be decomposed into two parts: one is the basic stimuli and another is the metamerism black. The basic stimuli represent the tristimulus information of the color stimuli, while the tristimulus value of the metamerism black is $[0,0,0]$. This hypothesis means that one pair of metamerism has the same basic stimuli spectrum and the different metameirsm black spectrum. Thus the reproduction spectrum $s_{r}$ and the estimated spectrum $s$ ' should have the same basic stimuli, which ensures the output spectrum and the source can achieve color appearance matching. To obtain the spectral matching, the metamerism black of $s_{r}$ and the source spectral $s$ should be as approximate as possible.

Therefore, to achieve the metamerism correction function $M C(\square)$, we firstly use the Matrix-R [8-9] method to get the basic stimuli of estimated spectrum $s$ '. Defining a matrix $\boldsymbol{R}$ as

$$
\boldsymbol{R}=\boldsymbol{A}\left(\boldsymbol{A}^{T} \boldsymbol{A}\right)^{-1} \boldsymbol{A}^{T}
$$

Where $\boldsymbol{A}$ is the united matrix of illuminant spectrum and standard observer Color Matching Function and can be denoted as

$$
\boldsymbol{A}=k \square \operatorname{diag}\left(\boldsymbol{E}^{\prime}\right) \square \boldsymbol{F}
$$

The basic stimuli $s^{\prime \prime}$ of spectrum $s^{\prime}$ is the projection of $s^{\prime}$ on $\boldsymbol{R}$, that is

$$
\boldsymbol{s}^{*}=\boldsymbol{R} \boldsymbol{s}^{\prime}
$$

The metamerism black of the source spectrum $s$ can be calculated by

$$
\boldsymbol{b}=(\boldsymbol{I}-\boldsymbol{R}) \boldsymbol{s}
$$

where $\boldsymbol{I}$ is identity matrix. Thus, the ultimate reproduction spectrum is

$$
s_{r}=s^{*}+b
$$

\section{Experiments}

In order to validate the algorithm, two multispectral images are chosen in the experiments. The spectral reflectance of the images is sampled in $400 \mathrm{~nm}$ to $700 \mathrm{~nm}$ at $10 \mathrm{~nm}$ intervals. The dimension of the reflectance data are 31. The RGB images synthesized from the multi-spectral images are shown in Figure 2. Figure 2(a) is a typical skin image and Figure 2(b) is s scene picture with high color saturation. According to the requirement of CIECAM02 model, the parameters of the viewing condition about the source and the reproduction images are set as in Table 1, where $X_{w} Y_{w} Z_{w}$ is the tristimulus values of white of the illuminant. The surround is surroundings which includes Dark, Dim and Average. $L_{A}$ is the luminance of the 
adapting field and it can be set as $20 \%$ of white luminance. In the experiments, the illuminant in source scene is D65 and in reproduction scene is A. The surround is Average. The other parameters in CIECAM02 can be derived from the above three parameters.

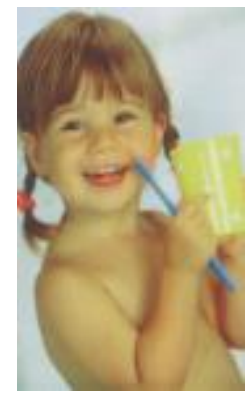

(a) Young-girl

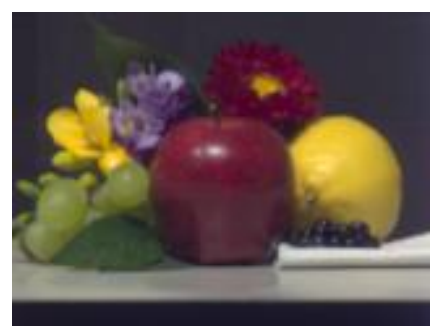

(b) Fruits-and-flowers

Figure 2. RGB Images Synthesized from Multi-Spectral Images

Table 1 Parameters of Viewing Condition

\begin{tabular}{ccc}
\hline Parameters & $\begin{array}{c}\text { Source viewing } \\
\text { condition }\end{array}$ & $\begin{array}{c}\text { Reproduction } \\
\text { viewing condition }\end{array}$ \\
\hline$X_{w} Y_{w} Z_{w}$ & $(95.05,100.00,108.88)$ & $(109.84,100.00,35.56)$ \\
surround & Average & Average \\
$L_{A}\left(c d / m^{2}\right)$ & 23.2 & 15.2 \\
\hline
\end{tabular}

The evaluation for the algorithm is done from spectral and color appearance precision. The IPT space [10-11] takes human visual characteristics and image space characteristics into account, so the evaluation for color appearance is implemented in this space. The chroma metric $\Delta I m$ of this space is used for color appearance error evaluation. It is $\Delta I m=\sqrt{\Delta I^{2}+\Delta P^{2}+\Delta T^{2}}$. The root-mean-square error equation $E_{R M S}$ [12-13] is used for spectral error evaluation. It is

$$
E_{\text {RMS }}=\sqrt{\frac{1}{N} \sum_{i=1}^{N}\left(\Delta \beta\left(\lambda_{i}\right)\right)^{2}}
$$

where $N$ is dimension number of the high-dimension spectral space. $\Delta \beta\left(\lambda_{i}\right)$ is the error of source spectrum and reproduction spectrum at the wavelength $\lambda_{i}$. Table 2 shows the comparison of color error with and without color appearance mapping. From Table 2 we know that the color error of the source and the reproduction image with color appearance mapping is much smaller than those without color appearance mapping. The color error of the former is almost impossible to be perceived by the human eyes. Table 3 shows the spectral error of the source and the reproduction spectral reflectance of the images. In the Table the spectral error is very small. Form this we know the reproduction spectra can well maintain the information of the source spectra.

Table 2. Color Error With and Without Color Appearance Mapping

\begin{tabular}{cllllll}
\hline \multirow{2}{*}{ Test image } & \multicolumn{3}{c}{ With color appearance mapping } & \multicolumn{3}{c}{ Without color appearance mapping } \\
\cline { 2 - 7 } & Mean $\Delta$ Im & MIN $\Delta$ Im & MAX $\Delta$ Im & Average $\Delta$ Im & MIN $\Delta$ Im & MAX $\Delta$ Im \\
\hline Fig.2(a) & 1.8208 & 0.4051 & 4.0612 & 19.2860 & 7.1546 & 38.7142 \\
Fig.2(b) & 2.4460 & 0.9922 & 5.3261 & 19.4990 & 7.3282 & 28.7884 \\
\hline
\end{tabular}


Table 3. Spectral Error

\begin{tabular}{cccc}
\hline Test image & Mean $E_{R M S}$ & MAX $E_{R M S}$ & MIN $E_{R M S}$ \\
\hline Fig.2 (a) & $8.1739 \times 10-4$ & 0.0041 & $1.3356 \times 10-5$ \\
Fig.2 (b) & $1.3356 \times 10-4$ & 0.0019 & $4.5899 \times 10-8$ \\
\hline
\end{tabular}

\section{Conclusions}

To solve the problem that the different viewing condition results in different color appearance between a reproduced image and its original during multi-spectral image reproduction, a new algorithm based on color appearance mapping for multi-spectral image reproduction is presented. By introducing the color appearance transformation at the source side and the inverse transformation at the destination side, the reproduction image matches the source image in color appearance in viewing-condition-independent space. Meanwhile, in order to maintain the spectral information in the source spectral image, the metamerism correction is implemented for the evaluated spectral reflectance based on the source spectral reflectance. The corrected spectra are used as the reproduction spectra. Experiments show that the new method achieves the color appearance matching in different viewing condition in multi-spectral image reproduction, at the same time it preserves the spectral information of the source image as more as possible.

\section{Acknowledgements}

Authors gratefully thank the co-workers for concerning the research project and also acknowledge the support of Science Foundation of Shaanxi province in China (Grant No. 2012JM8044), Research Fund of Shaanxi Provincial Education Department (Grant No. 12JK0733) and Research Fund of Xi'an University of Posts \& Telecommunications (Grant NO. 1504).

\section{References}

[1] R. Sitnik, J. Krzesłowski and G. Mączkowski, “Archiving shape and appearance of cultural heritage objects using structured light projection and multispectral imaging”, Optical Engineering, vol. 51, no. 2, (2012), pp. 021115-1.

[2] R. S. Berns and T. Chen, "Practical total appearance imaging of paintings", Proc. of IS\&T, Copenhagen, Denmark, (2012) June, pp. 162-167.

[3] M. Bouzid, A. Khalfallah, A. Bouchot, M. S. Bouhlel and F. S. Marzani, "Automatic cell nuclei detection: a protocol to acquire multispectral images and to compare results between color and multispectral images", Proc. SPIE, Imaging, Manipulation, and Analysis of Biomolecules, Cells, and Tissues, San Francisco, California, USA, (2013) February 02, pp. 85871J.

[4] D. Jakovels, I. Kuzmina, A. Berzina, L. Valeine and J. Spigulis, "Noncontact monitoring of vascular lesion phototherapy efficiency by RGB multispectral imaging", Journal of Biomedical Optics, vol. 18, no. 12, (2013), pp. 126019.

[5] M. D. Fairrchild, "Color Appearance Models", $2^{\text {nd }}$ Ed. John Wiley \& Sons, (2005).

[6] N. Moroney, M. D. Fairchild, R. W. G. Hunt, C. Li, M. R. Luo and T. Newman, "The CIECAM02 color appearance model", Proc. Of IS\&T/SID $10^{\text {th }}$ Color Imaging, Scottsdale, Arizona, USA, (2002) November 1215, pp. 23-27.

[7] G. Wyszechi and W. Stiles, "Color Science”, $2^{\text {nd }}$ Ed. John Wiley \& Sons, (1982).

[8] B. J. Cohen and E. W. Kapauf, "Metameric color stimuli fundamental metamers and Wyszecki's metameric blacks", Am. J. Psychol, vol. 95, (1982), pp. 537-564.

[9] Y. H. Zhao and R. S. Berns, "Image-based spectral reflectance reconstruction using the Matrix R method",Color Research and Application, vol. 32, no. 5, (2007), pp. 343-351.

[10] M. D. Fairchild and G. M. Johnson, "iCAM framework for image appearance, differences and quality", Journal of Electronic Imaging, vol. 13, no. 1, (2004), pp. 126-138. 
[11] G. M. Johnson, X. Song, E. D. Montag and M. D. Fairchild, "Derivation of a color space for image color difference measurement", Color Research and Application, vol. 35, no. 6, (2010), pp. 387-400.

[12] H. I. Francisco, R. Mitchell and R. S. Berns, "Comparative study of metrics for spectral match quality", Proc. of the First European Conf. on Color Graphics, Imaging, and Vision, Poitiers, France, (2002) April, pp.492496.

[13] J. Conde, H. Haneishi, M. Yamaguchi, N. Ohyama and J. Baez, "CIE-XYZ Fitting by Multispectral Images and Mean Square Error Minimization with a Linear Interpolation Function", Revista Mexicana De Fisica, vol. 50, no. 6, (2004), pp. 601-607.

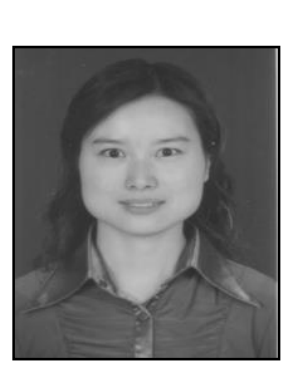

\section{Authors}

Ying Wang, she was born in 1977. She is a lecturer of School of Computer Science \& Technology, Xi'an University of Posts \& Telecommunications. She received her Ph.D. degree majoring in Computer Architecture from School of Computer Science \& Technology, Xidian University. Her research interests on color science and multispectral image input/output technology.

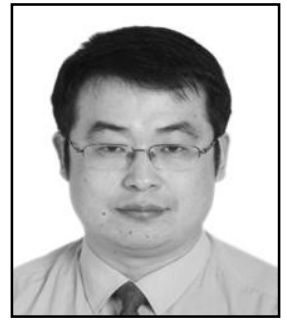

Sheping Zhai, he was born in 1971. He is an associate professor and vice dean of School of Computer Science \& Technology, Xi'an University of Posts \& Telecommunications. He received his Ph.D. degree majoring in Computer Science and technology from School of Electronics and Information Engineering, Xi' an Jiaotong University. His research interests on intelligent information processing and Semantic Web and Service computing.

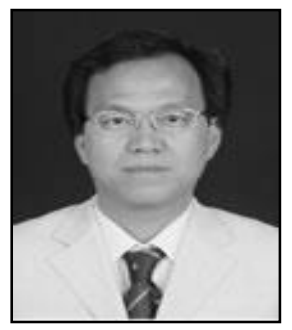

Zhongmin Wang, he was born in 1967. He is a professor and dean of School of Computer Science \& Technology, Xi' an University of Posts \& Telecommunications. He received his Ph.D. degree from Beijing Institute of Technology. His research interests on intelligent information processing, robot technology and computer vision. 